\title{
Sources of errors by capuchin monkeys on delayed response
}

\author{
JAMES L. LENTZ and JAMES E. KING \\ University of Arizona, Tucson, Arizona 85721
}

\begin{abstract}
Six capuchin monkeys were trained on a series of indirect, delayed response problems with delay intervals ranging from 0 to $60 \mathrm{sec}$. Data were analyzed by sequential state theory in order to quantify random and nonrandom components of the subjects' response sequences. A tendency of the subjects to repeat responses to the position chosen on the previous trial was a significant source of proactive interference even when effective orienting responses were elicited by the predelay cue. Changes in random and nonrandom response components with increasing delays suggested a two-phase process: the first for delays up to 7 sec characterized by increases in random and nonrandom error-producing responses, and the second for delays greater than $7 \mathrm{sec}$ characterized by increases in random responding only.
\end{abstract}

Sequential state theory (SST) is a method of quantifying both random and systematic sources of errors in two-choice, discrete trial data (King \& Fobes, in press). Although this method is similar to Levine's (1965) hypothesis analysis, it differs in two important respects. Whereas Levine's method requires the assumption that hypotheses occur in three trial units and that subjects do not switch hypotheses within these units, SST incorporates the less restrictive assumption that response states (analogous to hypotheses) can be defined in terms of only one or two trial units. The second important difference between the two methods is that SST permits simultaneous measurement of both sequentially dependent and sequentially independent states. A sequentially dependent response state is one that is defined in terms of some event occurring on the previous trial. One example is the tendency to choose the position rewarded on the previous trial (State F). Conversely, response states totally independent of events on preceding trials will be designated as sequentially independent and are exemplified by random responding (State $R$ ) and by position habits or position bias (State B).

The ability of SST to measure the relative contributions of a variety of sequentially dependent errorproducing states makes it especially suited for the measurement of error types on the delayed-response task. SST is particularly suited to answer questions about the role of intra- and intertrial determinants of delayed response performance.

In a previous experiment designed to discern whether delayed-response errors occur as a function of intra- or intertrial effects, Fletcher, Garske, Barron,

This research was partially supported by Training Grant MH11286 from the U.S. Public Health Service. Requests for reprints should be sent to Dr. James E. King, Department of Psychology, University of Arizona, Tucson, Arizona 85721. and Grogg (1968) measured intertrial effects by comparing the performance of rhesus monkeys when the baited positions on Trials $\mathrm{N}$ and $\mathrm{N}-1$ were the same or different. An additional comparison was made between normally baited trials (using both the direct and indirect method) and pseudotrials in which no predelay cue was presented. The monkeys manifested a preference for the position baited on the previous trial only when they failed to establish an effective orienting response to the predelay cue. Fletcher et al. consequently argued that intertrial information is a source of delayed-response errors only in the absence of an effective orienting response. Using somewhat similar tests for assessing proactive effects on a delayed-matching-from-sample task, Moise (1976) found no difference in performance when the matched positions on Trials $\mathrm{N}$ and $\mathrm{N}-1$ were the same or different but found higher performance on Trial $N$ when the nonspatial sample stimulus was the same on both trials.

The method used in the present paper offers some distinct advantages over the methods used previously. Since Fletcher et al. examined performance on Trial $\mathbf{N}$ when the baited position on Trial $\mathrm{N}-1$ was either the same as or different from that on Trial $\mathbf{N}$, the measure obtained was a confounded mixture of winstay lose-shift with respect to position (State F) and perseveration (State $P$ ). Similar problems affected Moise's measures of the effect of correct response position. The SST technique used in the present study allowed us to make unconfounded measures of these response tendencies. Application of SST to delayedresponse data allows determination of the change in the pattern of response states as delay intervals are increased to the point of near chance level performance. A basic question is whether these state changes parallel state changes in discrimination learning as the relevant stimuli became less and less discriminabile. 
The analogy between delayed response and discrimination among highly similar stimuli is not necessarily inappropriate if delayed response is viewed as a process of detecting traces of the predelay cue, which becomes more and more indistinct with increasing delays (a trace decay theory of this type has been proposed for delayed matching by Roberts \& Grant, 1976).

King and Fobes (in press) plotted the pattern of state probability changes in capuchin monkeys trained on a simultaneous brightness discrimination. With these highly adapted animals, as the difference between the positive and negative stimuli decreased, random responding increased while detect or correct responding decreased. Consequently, the sum of random and correct responding remained constant. All other systematic response tendencies (viz., simple position preference and various sequential dependencies) also remained constant across all levels of problem difficulty. Furthermore, the subjects' detect probabilities on the easiest problem were positively correlated with random responding on the most difficult (impossible) problem.

The study described here was addressed to the two general issues described above. First, the importance of intra- and intertrial error sources was determined by measuring the effects of two basic potential sources of proactive (intertrial) interference-namely, position of reward and position of the subject's response on the previous trial. The hypothesis of Fletcher et al. (1968) would be substantiated if these two error sources made no significant contribution to total errors under conditions in which an initial orienting response to the positive locus was effectively elicited.

Second, the pattern of response state changes accompanying increased delays was determined. A difference between these changes and those previously observed by King and Fobes (in press) following an increase in the difficulty of brightness discriminations would indicate a qualitatively different mechanism mediating the two types of problems.

\section{METHOD}

\section{Subjects}

Four male and two female capuchin monkeys (Cebus apella) were subjects. These animals had received extensive practice on learning set and delayed response problems and were from the group used by Scanlon and King (1976) on a modified samenessdifference learning set concept. Throughout testing on the current experiment, these animals were maintained at $87 \%-93 \%$ of their ad-lib weights and were fed once daily after testing.

\footnotetext{
Apparatus

Testing was performed manually in a modified version of the Wisconsin General Test Apparatus (WGTA), which was equipped with both a forward opaque screen and a one-way mirror screen. The stimulus presentation apparatus consisted of a four-chambered box with $7.5-\mathrm{cm}^{2}$ translucent panels $2.5 \mathrm{~cm}$ apart (edge to edge) facing the subject. Thus, the subject faced a $1 \times 4$ matrix of stimulus panels; however, for this experiment, a black Lucite mask covered the outer two panels, making only the center two doors available. The translucent Lucite panels were hinged at the top
}

so that the subject could respond by pushing the panel in and retrieving the reinforcer ( $1 / 4$ miniature marshmallow) placed on a small white circle in the center of the chamber. The correct position on a given trial was signaled by illuminating the stimulus panel for $5 \mathrm{sec}$. When the subject made a correct response (viz., to the previously illuminated panel), movement of the stimulus panel $.25 \mathrm{~cm}$ closed a microswitch, illuminating the chamber behind the panel and providing a secondary reinforcer. When an incorrect response was made, the chamber remained dark.

Interposed between the stimulus panels and the subject was the one-way screen, which was raised only at the end of each delay interval. Subjects could thereby view the lighted panels through the one-way screen but were prevented from touching either panel until the termination of the delay interval. After the subject pushed the panel and retrieved any marshmallow present, the one-way screen was lowered. The intensity of the light in the test cage was adjusted so that the two stimulus doors were visible through the mirror screen only when one of them was illuminated. Thus, upon offset of the cue light, the subject was unable to maintain visual contact with the panel. Delay (intratrial) intervals and intertrial intervals $(15 \mathrm{sec})$ were timed manually by the tester with a foot-pedal-actuated clock.

\section{Procedure}

Twelve 24-trial stimulus sequences used throughout testing and adaptation were constructed with the following constraints: each of eight possible three-trial right-left triads (RRR, RRL, RLR, RLL, LRR, LRL, LLR, and LLL) occurred once on each 24 trial sequence, and the same side was not baited on more than three consecutive trials. One of these 24-trial stimulus sequences was presented daily to each of the subjects and the ordering of the presentation of the stimulus sequences was randomized across days.

The subjects were first trained to discriminate between an illuminated and a nonilluminated door to retrieve a reinforcer (one-way screen up). After a subject had responded correctly for at least 21 of 24 trials on 2 consecutive days, the 0 -sec delay condition, the subjects received 60 days' $(1,440$ trials) practice with a 2 -sec-delay condition (one-way screen raised $2 \mathrm{sec}$ after cue offset). During this 2-sec adaptation phase and the subsequent experimental phase, each day's test trials were preceded by two priming trials (one for each side). A priming trial consisted of presenting the cue light with the one-way screen down, raising it $1 \mathrm{sec}$ before cue offset, and allowing the subject to retrieve a marshmallow fragment from the lighted locus.

After the extensive practice on the 2-sec-delay condition, the subjects were tested for 72 days with 24 trials presented each test day. During this testing, all subjects received 288 trials on each of six delay conditions. The delays were $0,1,3,7$, or $60 \mathrm{sec}$. In addition, pseudoproblems in which the predelay cue presentation did not occur were presented and the one-way screen was raised $5 \mathrm{sec}$ after the beginning of the trial. These six delay conditions were presented in three trial problems administered randomly with the following constraints: (1) no delay condition followed itself; (2) 60-sec-delay problems and pseudoproblems were never presented sequentially; (3) each delay condition was presented under all eight possible three-trial right-left sequences within a 6-day period; and (4) each delay interval was used at least once and no more than four times in a given 2-day test period. Thus, on most test days, all six conditions were presented at least once.

\section{Data Analysis}

The data for each subject were analyzed by the SST technique for each of the six delay conditions. Probabilities of the following six response states were determined.

(1) State D (detection) arises from learning, successful retention, or any other process leading to above chance level of correct responding. This state is always manifested by a correct $(t)$ response.

(2) State B (position preference) accounts for an overall difference in the frequency of left and right responses. It is manifested 
by a response to the favored side and is analogous to a sequence of outcomes obtained by successive throws of a biased or "loaded" coin.

(3) State $\mathbf{R}$ (random) results when responding is not governed by any systematic rule, and consequently all possible responses are equally likely to occur when this state is in effect. States $D, B$, and $R$ are all sequentially independent in the sense that they are not defined in terms of the responses or outcomes of preceding trials. However, all of the remaining states measured did entail a sequential dependency from the immediately preceding trial.

(4) State $P$ results in above chance perseverations of the same position response on two successive trials. The distinction between manifestations of State P and State B is important because both are frequently described as "position preference" or "perseveration." The perseveration hypothesis in Levine's (1965) analysis is, in fact, a composite of State $\mathbf{P}$ and State $B$ responding. Position perseveration from State $P$, by definition, includes equal numbers of left and right perseverations. Thus, a response sequence yielding a high probability of State $\mathbf{P}$ would include relatively large numbers of both left-left and right-right response sequences, in contrast with a response sequence with a high probability of State B that would simply contain a large preponderance of either left or right responses.

(5) State F (win-stay, lose-shift with respect to position) occurs when responding is sequentially dependent upon the position of the reward on the preceding trial. For example, one possible manifestation of State $F$ would be a response to the left position on Trial $\mathbf{N}$ when the subject had correctly responded to the left position on Trial $\mathbf{N}-1$ (win-stay). Another manifestation of this state would be a response to the left on Trial $\mathrm{N}$ when the subject had incorrectly responded to the right stimulus position on Trial N-1 (lose-shift).

(6) State $S$ (outcome clustering) occurs when two successive correct responses and two successive incorrect responses are more frequent than would be expected on the basis of the overall probability of correct responding.

The technique for estimating state probabilities requires categorizing successive three-trial sequences into one of 32 types based on the eight possible correct-incorrect sequences and the four possible response sequences, dependent upon whether the Trial 2 and Trial 3 position responses are the same as or different from the Trial 1 response (Levine's procedure is based on a similar categorization). SST, unlike Levine's technique, considers Trials 2 and 3 separately, so that the state governing Trial 2 may be the same as or different from the state governing Trial 3 . The SST procedure requires solution of 16 simultaneous linear equations in a manner similar to Levine's Method 2. However, the overall probability estimates for the above six states can be expressed by the following equations, where $D, B, P, F, S$, and $R$ refer to the corresponding state probabilities. $C_{0}$ is the observed proportion of correct responses; $A_{0}$ and $B_{0}$ are the observed proportions of the most and least frequent position responses, respectively; $P_{0}$ is the observed proportion of position perseverations (right-right or left-left); $F_{o}$ is the observed proportion of responses to the position that was correct on the previous trial; and $S_{0}$ is the observed proportion of positive-positive or negativenegative trial outcomes. DF is referred to as bistate probability and is the probability of State D and State F each occurring once on Trials 2 and 3 in any order. DP is similarly defined, while $D_{2}$ is the probability of State D occurring on both Trials 2 and 3. DS, however, is the probability of State D occurring on Trial 2 and State S occurring on Trial 3.

$$
\begin{aligned}
& D=2 C_{o}-1 \\
& B=2 A_{o}-1 \\
& P=2 P_{o}-1-\left[A_{o}^{3}-B_{o}^{3}\right]+1 / 3\left[A_{o}^{2} B_{0}-A_{0} B_{0}^{2}\right]-1 / 4 D F
\end{aligned}
$$

$$
\begin{aligned}
& F=2 F_{o}-1-1 / 4 D P \\
& S=2 S_{o}-1-1 / 2 D_{2}+1 / 2 D S \\
& R=1-D-B-P-F-S
\end{aligned}
$$

Values of DF, DP, $D_{2}$, and DS can only be determined after solving the entire set of 16 linear equations. That procedure was used to obtain the values reported below. However, if these bistate probabilities are simply eliminated from the above equations, a fairly close approximation can easily be obtained.

Two composite statistics based on $D$ and $R$ were also analyzed. The first of these, $\mathbf{A}$, or attention, is defined as $\mathbf{D}+\mathbf{R}$. $\mathbf{A}$ is an attentional measure in the specific sense that it estimates the probability that subjects' responses are not generated by systematic error-producing biases. Inclusion of randomness as a component of attention is no doubt somewhat counterintuitive. However, if randomness is indeed an index of resistance to systematic errorproducing response tendencies, then our definition of $\mathbf{A}$ becomes more reasonable. This point will be addressed further in the Discussion.

The second composite statistic is $\mathbf{K}$, the conditional probability of detection given that the subject is attending or not manifesting a systematic response bias $[K=D /(D+R)] . K$, then, is a purified performance measure with all nonattentional biases and sequential dependencies parceled out.

\section{RESULTS}

To determine the response components accounting for significant portions of the monkeys' responses, $t$ tests were used to ascertain which mean state probabilities over all conditions differed significantly from zero. Mean probabilities of detect (D), random $(\mathrm{R})$, simple position bias (B), position perseveration (P), and outcome clustering (S) were all significantly greater than zero, while win-stay lose-shift for position (F) was not. The mean values and significance levels are depicted in Table 1.

During the last 5 test days of the 2-sec adaptation period, the mean percentage of correct responses was $78 \%$. In the test phase, the mean percentage of correct responses decreased significantly as the delay interval increased $[F(5,25)=44.85, p<.001]$ and ranged from $86 \%$ with the 0 -sec delay to $54 \%$ with the 60 -sec delay.

Figure 1 shows that most errors resulting from increased delays were attributable to random responding (State R), which increased significantly as a function

Table 1

Mean State Values

\begin{tabular}{cc}
\hline State & Mean \\
\hline D & $.3344^{* *}$ \\
R & $.2338^{* *}$ \\
B & $.1544^{*}$ \\
P & $.1344^{*}$ \\
S & $.0659^{*}$ \\
F & $.0098^{\circ}$ \\
\hline
\end{tabular}

Note-Significance levels are based on two-tailed t tests (df $=5)$. ${ }^{*} p<.002 . \quad{ }^{* *} p<.02 . \quad$ tNonsignificant. 


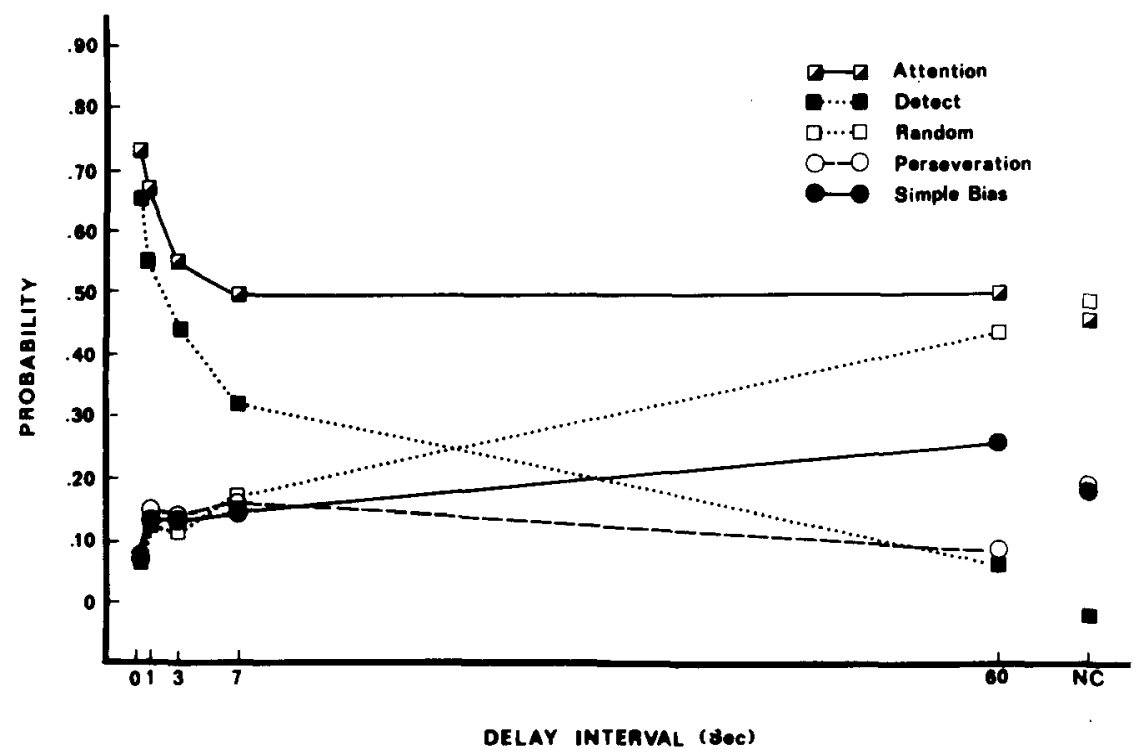

Figure 1. Probabilities of various state strengths as a function of delay interval.

of delay interval $[F(5,25)=13.23, p<.001]$. Probabilities of all remaining error-producing states $(B, P$, $\mathrm{F}$, and $\mathrm{S}$ ) did not change significantly as a function of delay. However, the previously described composite state probabilities did vary significantly over delays. Composite measures that decreased significantly with delay intervals were attention $(D+R)$, a measure of the extent to which responding is free of systematic response biases $[F(5,25)=2.92, p<.04]$, and $K[D /$ $(D+R)]$, a conditional measure of correct responding given that no systematic response bias occurs $[F(5,25)=30.59, p<.01]$. Conversely, the summed probability of position perseveration $(\mathrm{P})$ and position bias (B) increased significantly over delays $[F(5,25)$ $=3.02, \mathrm{p}<.03$ ].

Since Figure 1 indicated a discontinuity in attention $(D+R)$ at $7 \mathrm{sec}$, two further series of analysis of variance tests were made, one for delay intervals from 0 to $7 \mathrm{sec}$ and the other from 7 to $60 \mathrm{sec}$. The decrease in detect probabilities (D) was significant between 0 and $7 \mathrm{sec}[\mathrm{F}(3,25)=13.28, \mathrm{p}<.001]$, as well as between 7 and $60 \sec [F(1,25)=19.49, p<.001]$. Conversely, a concomitant increase in random responding responding (R) occurred over both of these same intervals $[F(3,25)=9.06, p<.001$, between 0 and 7 sec; $F(1,25)=13.63, p<.001$, between 7 and $60 \mathrm{sec}]$. However, the decrease in $D$ and the increase in $\mathbf{R}$ were not symmetrical; from 0 to $7 \mathrm{sec} D$ decreased faster than $\mathbf{R}$ increased, thereby resulting in a decrease in attention $(D+R)$ between 0 and $7 \mathrm{sec}$. Attention was virtually constant between 7 and $60 \mathrm{sec}$.

Although, as noted before, the sum of the positionrelated biases $(P+B)$ increased significantly between 0 and $60 \mathrm{sec}$, it was constant between 7 and $60 \mathrm{sec}$. Thus, the increase in position-related biases was restricted to delays between 0 and 7 sec.
Comparison of state strengths on the pseudotrial and 60 -sec-delay conditions showed whether elimination of the predelay cue elicited response biases in strengths different from those elicited by a delay of sufficient length to cause correct responding near change level. Figure 1 shows that differences between the two conditions were not striking. Random responding accounted for approximately $50 \%$ of the responses in both conditions. Win-stay lose-shift for position (State F) was the only state with significantly different values in the two conditions $[t(5)=3.27$, $\mathrm{p}<.05]$, and the absolute magnitude of the difference was small $[F=-.005$ and .07 for the $60-\mathrm{sec}$ and pseudotrial conditions, respectively].

To assess the adequacy of the SST procedure, the observed frequency for each of the 32 types of threetrial sequences was compared with that predicted by the bistate probabilities from the analysis. The statistic used was almost identical to what Levine (1965) referred to as proportion of variance explained, or PVE.

$$
\mathrm{PVE}=1-\frac{\sigma_{\mathrm{p}}^{2}}{\sigma_{\mathrm{o}}^{2}},
$$

where $\sigma_{\mathrm{p}}^{2}$ is the mean squared deviations between the 32 observed frequencies and the frequency expected based on all the bistate values. The other variance, $\sigma_{\mathrm{O}}^{2}$, is the mean squared deviations between the observed frequencies and the overall mean frequency. The PVE yields a measure of the proportion of the total variation between observed frequencies accounted for by the measured states and is thus comparable to an $\mathrm{r}^{2}$ value. An overall PVE was calculated for each subject, based on data from all delay conditions; the 
obtained PVE values ranged from .763 to .937 , with a mean of .811. (This corresponds to a correlation of .900 between observed and expected frequencies of three-trial sequences.)

\section{DISCUSSION}

The first question this study was designed to answer was which type of intertrial interference accounts for most responses in delayed response. The results showed that position perseveration (State P) accounted for about $13 \%$ of the responses, while virtually none were accounted for by win-stay lose-shift for position (State F). Thus, intertrial interference based on the position of the subject's response on the previous trial is clearly stronger than that based on the position that was correct on that previous trial. This may reflect greater durability of the memory for responses (position perseveration) than for the memory of rewarded position (win-stay lose-shift responding). This moderately large value for $P$ may also have been mediated, at least in part, by the monkeys' frequent continuation of their physical orientation to the position previously chosen, regardless of the outcome of that choice.

Win-stay lose-shift responding was more frequent in the pseudotrial condition than in the 60-sec-delay condition; position perseveration, however, was not significantly different in the two conditions. These two results suggest that errors based on memory of the previously rewarded position are more effectively eliminated by the predelay cue than are errors based on memory of the previous response.

A continuing problem in the history of delayed response and delayed matching has been the question of the importance of intertrial or proactive interference as a source of errors (Fletcher et al., 1968; Gleitman, Wilson, Herman, \& Rescorla, 1963; Medin \& Davis, 1974; Moise, 1976; Yerkes \& Yerkes, 1928). Fletcher et al. emphatically argued that well-trained monkeys' use of intertrial information in delayed response occurs only when an effective orientation toward the positive locus is not elicited by the predelay cue.

Therefore, intrusion of response biases established by or already present on previous trials should be a consequence, not a cause, of an ineffective predelay cue. Data from the study described here that are most relevant to Fletcher's hypothesis are the strengths of simple position bias (State B) and position perseveration (State P) on the pseudotrials and on the conventional delay trials. If, as the Fletcher hypothesis suggests, these position-related biases were manifested only on trials for which no effective orientation was elicited by the predelay cue, then $P$ and $B$ should have been higher on pseudotrials than on conventional delay problems for which the probability of correct responding at 0 -sec delay exceeded chance. The above prediction is made because effective orientation could never occur on pseudotrials but would occur on a large proportion of conventional delay trials (estimated by $D$ for 0 -sec delay). Hence, susceptibility to intertrial biases would be restricted to a proportion $1-\mathrm{D}$ of the conventional trials but would be present on all pseudotrials.

Yet, neither P nor B, nor their sum, was significantly higher on pseudotrials than on any of the conventional delay conditions. The implication of this result is that both of the intertrial position-related biases are indeed sources of errors in spatial delayed response, even on some trials for which an effective orienting response was elicited by the predelay cue. These biases are not simply consequences of ineffective initial orientation to the positive locus.

The second major question to which this study was addressed is whether the increase in errors with lengthening delay intervals results entirely from increases in the random component of the response sequences. King and Fobes (in press) found that as the difficulty of brightness discriminations was increased, increased random responding accounted for almost all of the increased errors. In the present study, attention or $D+R$, instead of remaining constant as in the King and Fobes discrimination study, decreased from delays of 0 to $7 \mathrm{sec}$. The decrease in $D+R$ was accompanied by increases in position-related responding $\mathbf{P}+\mathbf{B}$. Thus, imposition of delays effectively elicited a process akin to spontaneous recovery for position-related states, whereas rendering a discrimination difficult by use of similar discriminative stimuli did not.

A further indication of a basic difference between the state-eliciting properties of discrimination and delayed response was shown in the correlation between $D$ and $R$. Whereas King and Fobes reported a significant positive correlation $(r=.89)$ across their capuchin monkeys between $\mathrm{D}$ on the easiest discrimination and $\mathbf{R}$ on an impossible problem, the present delayed-response data revealed nonsignificant negative correlations between $\mathrm{D}$ on the $\mathbf{0}$-sec delay and $\mathrm{R}$ on the $60-\mathrm{sec}$ delay and pseudotrials $(r=-.77$ and -.60 , respectively). In other words, for delayed response, unlike brightness discrimination, high $\mathbf{R}$ on difficult or impossible problems does not predict high $\mathrm{D}$ on easier problems. This suggests that in the delayedresponse problem, initial orientation to the positive locus that affects performance on 0 -sec delay is independent of another memory-related mechanism that determines how effectively the information from the predelay cue is retained over long delays.

Examination of the state probabilities in Figure 1 suggests that intratrial forgetting followed two distinct phases. The first was from 0 - to 7-sec delays and was characterized by increases in the sum of all biased responding and an increase in random responding, while the second was from 7- to 60-sec delays and was characterized by increases in random responding 
alone, with biases remaining constant. This same information was alternatively depicted by a sharp decrease in attention $(D+R)$ or the probability of nonbiased responding from 0 - to 7 -sec delays followed by constant attention from $7-$ to 60 -sec delays. Thus, two processes or phases may be present in the performance loss caused by increasing delays. The first is a short-term process lasting for approximately $7 \mathrm{sec}$, which is governed by both inter- and intratrial sources of forgetting and manifested by increases in response biases as well as random responding, respectively. The second or long-term process occurs after $7 \mathrm{sec}$, is governed by intratrial sources of forgetting, and is manifested by a continuous increase in random responding, with attention remaining constant.

The results of this study emphasized the role of random responding, first, because of the large $\mathbf{R}$ values for the longer delay intervals and, second, because of the inclusion of $R$ as a component of attention. The importance of random responding is often deemphasized (e.g., Levine, 1965, 1974), since randomness measures were usually small and it was assumed that randomness was an artifact of some nonrandom choice behavior not explicitly measured. Indeed, systematic response tendencies present in the data but not measured by SST would have a complex effect, usually including a spurious increase in $\mathbf{R}$. Such unmeasured systematic responding would have two predicted effects: low PVE values and a significant negative correlation across subjects between PVE and R. In fact, PVE values were high (mean $P V E=.81)$ and the correlation between PVE and $R$ was small and nonsignificant $(r=-.20)$. Therefore, we argue that $R$ in highly trained subjects is a representative measure of true random responding.

Of course, any finite response sequence can be completely described by some response rule if there is no limit to the complexity of the rule. However, we believe it is highly unlikely that any one systematic error source more complex than those measured here accounted for more than a negligible number of responses. The definition of attention as $D+R$ is clearly unconventional and needs some justification. Any attentional measure must be specific to a particular stimulus dimension. Furthermore, in any learning or memory task, a reasonable assumption is that the greater the subjects' choices' correlation with irrelevant dimensions, the less the attention to the relevant dimension. Therefore, probabilities of nonattentional states $\mathrm{B}, \mathrm{P}, \mathrm{F}$, and $\mathrm{S}$ should be inversely related to attention. Even complete attention to the relevant dimensions will not result in totally correct responding whenever the delay interval is long enough to cause errors. Consequently, attention that does not result in State D can result only in random responding, since anything else would involve systematic responding that is evidence of nonattention (see King \& Fobes, in press).

\section{REFERENCES}

Fletcher, H. J., Garske, J. P., Barron, T., \& Grogg, T. M. Intertrial and intratrial determinants of delayed response of monkeys. Journal of Comparative and Physiological Psychology, $1968,65,66-71$.

Gleitman, H., Wilson, W. A., Jr.; Herman, M. M., \& Rescorla, R. A. Massing and within delay position as factors in delayed-response performance. Journal of Comparative and Physiological Psychology, 1963, 56, 445-451.

King, J. E., \& Fobes, J. L. Application of sequential state theory to complex learning and sensory discrimination. In S. J. Suomi \& L. A. Rosenblum (Eds.), Advances in the study of primate social behavior. New York: Academic Press, in press.

Levine, M. Hypothesis behavior. In A. M. Schrier, H. F. Harlow, \& F. Stollnitz (Eds.), Behavior of nonhuman primates (Vol. 1). New York: Academic Press, 1965.

Levine, M. Interpreting inconsistent response patterns: A reaction to Williams' estimate of "true hypothesis behavior." Journal of Experimental Child Psychology, 1974, 18, 369-371.

Medin, D. L., \& Davis, R. T. Memory. In A. M. Schrier \& F. Stollnitz (Eds.), Behavior of nonhuman primates (Vol. 5). New York: Academic Press, 1974.

Moise, S. L., JR. Proactive effects of stimuli: Delays and response position during delayed matching from sample. Animal Learning \& Behavior, 1976, 4, 37-40.

Roberts, W. A., \& Grant, D. S. Studies of short-term memory in the pigeon using the delayed matching-to-sample procedure. In D. L. Medin, W. A. Roberts, \& R. T. Davis (Eds.), Processes of animal memory. Hillsdale, N.J: Erlbaum, 1976.

Scanlon, J. L., \& King, J. E. Learning and transposition of an extended sameness-difference concept by slow and fast learning capuchin monkeys. Animal Learning \& Behavior, 1976, 4, 308-312.

Yerkes, R. M., \& Yerkes, D. N. Concerning memory in chimpanzees. Journal of Comparative Psychology, 1928, 8, 237-271.

(Received for publication April 6, 1980; revision accepted October 28,1980 .) 